\title{
Expertengespräch: Pädiatrie
}

Viele Eltern wünschen sich für ihre Kinder eine «sanfte» Medizin und stehen komplementärmedizinischen Behandlungsmethoden sehr offen gegenüber. Auch die Kinderärzte sind häufig offen für einen ganzheitlichen Ansatz. Bei akuten lebensbedrohenden Problemen ist jedoch die Schulmedizin gefragt: Dies ist der gemeinsame Nenner des Expertengesprächs zum Thema Pädiatrie mit Heiner Frei, Bernhard Wingeier und Silvio Schaller.

Welche sind die häufigsten Probleme bei Kindern, die Sie mit Ihren Methoden der Komplementärmedizin behandeln?

Frei: Wir machen in unserer Praxis die übliche kinderärztliche Grundversorgung, mit dem Unterschied, dass ca. $80 \%$ der Behandlungen homöopathisch sind.

Wingeier: Am häufigsten behandeln wir Infekte der oberen Luftwege, Mittelohrentzündungen, fieberhafte Infekte, Asthma und Neurodermitis. Bei allen Erkrankungen setze ich die Anthroposophische Medizin in irgendeiner Form ein. Bei Bedarf auch in Kombination mit der «Schulmedizin».

Schaller: In meiner Praxis für chinesische Arzneitherapie sind es Erkältungsgefahr bzw. gehäufte Infekte der oberen Luftwege wie Tonsillitis/ Pharyngitis oder Bronchitis mit Folgeproblemen wie chronischem TubenMittelohr-Katharrh, ausserdem Gehörprobleme, Nasenpolypen, Sinusitis, Allergien sowie akute und chronische Verdauungsprobleme.

Welchen Stellenwert haben aus Ihrer Sicht komplementäre Methoden in der Kinder- und Jugendmedizin?

Schaller: Jedes medizinische System hat seine Stärken und Schwächen. Im Bereich der Akutmedizin, der Traumatologie, muss die Medizin stark und schnell wirken. Im Bereich der chronischen, chronisch-rezidivierenden und funktionellen Erkrankungen spielen konstitutionelle, individuelle Faktoren eine wichtige Rolle. Hier ist z.B. die Chinesische Medizin sicher eine gute Alternative, oder besser: eine gute Ergänzung zur Schulmedizin.

Wingeier: Sie haben aus meiner Sicht einen hohen Stellenwert. Viele Eltern wünschen sich für ihre Kinder eine «sanfte» Medizin und sind sehr offen gegenüber einer komplementären Medizin. Sie möchten aber häufig gleichzeitig die Sicherheit der Schulmedizin und nicht selten auch deren Geschwindigkeit. Die Anthroposophische Medizin ist keine Alternative zur Schulmedizin. Die Schulmedizin ist ein Teil unserer Medizin und kommt dort zum Tragen, wo sie notwendig und gefragt ist. Die Methoden ergänzen sich.

Frei: In vielen Fällen - nicht in allen sind komplementäre Methoden mindestens ebenbürtig, oft sogar überlegen, wenn der behandelnde Arzt seine Methode beherrscht.

\section{Wo geraten Sie mit Ihren Methoden} an Ihre Grenzen?

Frei: Bei akuten schweren Notfällen, z.B. einem Asthmaanfall mit Sauerstoffmangel, oder bei schweren Infektionen wie den Meningitiden. Auch chirurgische Notfälle wie das akute Abdomen können und sollen nicht komplementärmedizinisch behandelt werden.

Schaller: Dem schliesse ich mich an. Auch die Onkologie ist vor allem eine Zuständigkeit der Schulmedizin.

Wingeier: Grenzen stellen sich bei akuten schweren Notfällen, bei denen eine rasche Besserung der bedrohlichen Situation herbeigeführt werden muss, und wenn die Kräfte des Patienten schon massiv geschwächt sind. Dann setze ich die Anthroposophische Medizin unterstützend neben der Schulmedizin ein. Nicht selten ist dann auch eine Hospitalisation angezeigt.

Wann ist von einer komplementärmedizinischen Behandlung unbedingt abzuraten?

Frei: In lebensgefährlichen Notfallsituationen, auch bei Krankheiten, die eine Substitution erfordern, z.B. Diabetes mellitus oder Hypothyreose. Bösartige Tumoren sollten komplementärmedizinisch höchstens begleitend, d.h. neben der konventionellen Behandlung, angegangen werden.

Wingeier: In akuten, lebensbedrohenden Situationen nicht als Erst- und schon gar nicht als alleinige Massnahme. In der Kinderonkologie ist eine alleinige komplementäre Therapie nicht indiziert, eine anthroposophische Begleittherapie, z.B. mit Mistelpräparaten, aber von hohem Nutzen.

\section{KARGER}

Fax +49761 4520714 Information@Karger.d www.karger.com
(๑) 2011 S. Karger GmbH, Freiburg 


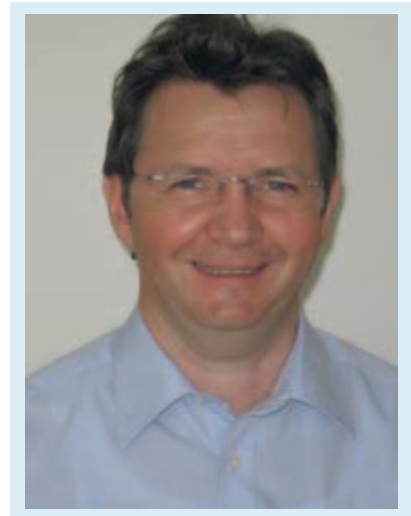

Dr. med. Bernhard Wingeier

(bernhard.wingeier@wegmanklinik.ch) ist Facharzt für Kinder- und Jugendmedizin; seit 1998 ist er in der Ita Wegman Klinik in Arlesheim (BL) tätig. Seine ambulante Sprechstundentätigkeit umfasst das ganze Gebiet der Kinderheilkunde, im Speziellen auch für anthroposophische Therapie bei allergischen Erkrankungen wie Asthma und Neurodermitis sowie onkologische Erkrankungen.

«Krankheit ist nicht nur etwas Negatives, sondern ein Teil des Lebens, mit dem wir uns auseinandersetzen müssen. Gesundwerden ist ein Prozess."

Was unterscheidet Sie als ganzheitlich praktizierenden Arzt von einem durch und durch «schulmedizinischen» Kollegen?

Wingeier: Der Zugang zum Kind, das Menschenbild und das Krankheitsverständnis aus der Anthroposophie heraus. Wir gehen weniger vom einzelnen Symptom als vielmehr vom ganzen Kind in seiner Umgebung aus. Auch sind wir der Überzeugung, dass Krankheit nicht nur etwas Negatives, sondern ein Teil des Lebens ist, mit dem wir uns auseinandersetzen müssen. Gesundwerden ist dann immer ein Prozess.

Schaller: Die Polarisierung in "ganzheitlich» und «schulmedizinisch» ist nicht sinnvoll; manchmal ist der Schulmediziner, manchmal der Komplementärmediziner in uns mehr gefragt. Sicher ist es so, dass die meisten chronischen Erkrankungen nicht mono- kausal auftreten und somit auch keine schnellen Lösungen verfügbar sind. Wir brauchen deshalb mehr Zeit zum Zuhören, Fragen und Untersuchen. Wir versuchen, Konstitution und Lebensstil in Analyse und Therapie einzubeziehen.

Frei: Ich versuche immer, den Patienten als Ganzes zu behandeln und mich nicht auf einzelne Symptome zu beschränken, wie das die konventionelle Medizin meist tun muss.

\section{Hat sich die Einstellung der eher klassisch arbeitenden Kollegen in der Pädiatrie zu Ihrem Ansatz in den letzten Jahren gewandelt?}

Frei: Ich habe den Eindruck, dass viele, vor allem jüngere Kolleginnen und Kollegen der Homöopathie gegenüber offener geworden sind. Sie sehen, dass man damit Probleme lösen kann, die sich konventionell nicht befriedigend lösen lassen, z.B. eine Infektanfälligkeit, häufige Kopf- oder Bauchschmerzen, allergische Erkrankungen usw.

Wingeier: Ja. Die Einstellung ist offener geworden; es besteht eine grössere Akzeptanz. Zum Teil werden Kinder auch für eine ergänzende anthroposophische Therapie von den Kollegen geschickt. Wichtig ist der gegenseitige Respekt; schliesslich bemühen sich auch diese Kollegen, für das Kind das Beste zu tun, einfach aus ihrer Sicht.

Schaller: Zwei wichtige Motivationen, sich für neues Wissen zu öffnen, sind einerseits das Bewusstsein um die eigenen Limitationen und andererseits die positive, praktische Erfahrung mit Neuem.

\section{Welche Vorteile hat Ihr Ansatz - und} welche Nachteile bringt er mit sich?

Frei: Die Vorteile der Homöopathie: Sie heilt schnell, sanft und verursacht keine Nebenwirkungen. Sie kann auch oft Krankheiten heilen, für die konventionell keine Therapiemöglichkeiten be- stehen. Ihre Nachteile: Homöopathie ist abhängig von einer genauen Symptom-Beobachtung durch die Eltern, und sie braucht etwas mehr Zeit, weil jedes Arzneimittel individuell bestimmt werden muss.

Schaller: Da Chinesische Medizin die konstitutionellen, individuellen Aspekte berücksichtigt, besteht die Möglichkeit, mehrere Behandlungsstrategien zu kombinieren. Die Wirkung ist oft nachhaltiger. Ein Nachteil im Fall der Arzneitherapie ist die unattraktive Galenik: Abkochungen oder Pulver mit intensivem Geschmack.

Wingeier: Der Vorteil der Anthroposophie besteht in einem anderen Zugang zum Kind, seiner Entwicklung und seiner Erkrankung. Ich habe eine grosse Palette an Heilmitteln und spezifischen Therapien. Es ist keine auf die Symptome beschränkte Medizin. Gerade bei den im Kindesalter häufigen banalen Infekten haben wir viele Möglichkeiten, den Selbstheilungsprozess des Kindes zu unterstützen. Allerdings braucht dies mehr Zeit und Geduld.

\section{Wirkt Ihr spezifischer Ansatz auch präventiv?}

Frei: Ja, ein richtig gewähltes homöopathisches Arzneimittel hat einen länger dauernden Schutz gegen verschiedene Krankheiten zur Folge. Wir haben das am Beispiel der Tonsillopharyngitiden bei Kindern zeigen können: Nach Behandlung einer akuten Erkrankung mit homöopathischen Einzeldosen (C 30) hatten die Kinder während den nächsten zwei Monaten signifikant weniger Infekte als danach.

Wingeier: Ja, in vielen Fällen. Dies ist eine besondere Stärke, aber nicht immer leicht zu erreichen.

Schaller: Die Chinesische Medizin hat eigentlich keine Strategien, um Symptome zu unterdrücken. Sie wirkt, indem sie Fehlfunktionen korrigiert, und sie wirkt somit auch präventiv. 


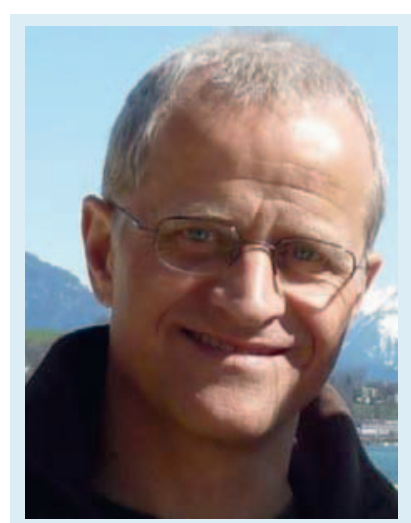

Pract. med. Silvio Schaller

(schallajia@gmx.ch) ist Allgemeinarzt und Mitinhaber einer Gruppenpraxis für Traditionelle Chinesische Medizin in Zürich. Seit 20 Jahren arbeitet er in seiner Praxis vorwiegend mit chinesischer Arzneitherapie. Rund die Hälfte seiner Klientel sind Kinder.

"Die Chinesische Medizin hat keine Strategien, um Symptome zu unterdrücken. Sie wirkt, indem sie Fehlfunktionen korrigiert, und somit auch präventiv.»

Wie entwickelt sich die Nachfrage nach Ihren Methoden?

Wingeier: Gut, steigend.

Schaller: Nach einer Boomphase ab Mitte der 90er-Jahre mit einem grossen Zuwachs an TCM-Therapeuten sehe ich heute eine gewisse Konsolidierung der Nachfrage. Jedes neu eingeführte Medizinsystem braucht eine gewisse Zeit, um sich zu etablieren und seinen Platz innerhalb des Systems zu finden.

Frei: Bei der Homöopathie ist die Nachfrage sehr gross; so gross, dass wir lange nicht alle Kinder behandeln können, deren Eltern zu uns kommen möchten. Es besteht auch ein Mangel an homöopathisch behandelnden Kinderärzten.

\section{Haben klassische Kinderkrankheiten wie Masern oder Mumps aus Ihrer Sicht einen biologischen Sinn?}

Schaller: Über Sinn und Unsinn von Krankheiten kann man gerne philosophieren. Jede schwere Komplikation bei Kinderkrankheiten ist eine zu viel. Jede Reise eines ungeimpften Kindes in ein Drittweltland mit Impfkampagne ist aus epidemiologischer Sicht ein Risiko. Ich befürworte deshalb die Kinderimpfungen.

Frei: Jede Krankheit kann bei einem Kind einen Entwicklungsschub auslösen, nicht nur Masern oder Mumps. Aber diese beiden Kinderkrankheiten sind wegen ihren Komplikationen nicht ungefährlich. Ich befürworte deshalb die Impfung.

Wingeier: Masern und Mumps sind Entwicklungskrankheiten. Sie können aus der Menschenkunde der Anthroposophie heraus verstanden werden. Sie ergeben aber nur dann einen Sinn, wenn sie auch richtig begleitet werden. Gerade Masern haben aber ein Komplikationsrisiko, vor allem bei ganz kleinen Kindern, bei chronisch kranken und geschwächten Kindern und ab der Pubertät. Darum müssen potenzieller Nutzen und Risiko genau gegeneinander abgewogen werden.

\section{Wie beurteilen Sie den Standard- impfplan: Sind alle Impfungen nötig?}

Frei: Die Basisimpfungen sicher. Man muss die Impffrage unbedingt von der Homöopathie abkoppeln. Impfgegnerschaft hat per se nichts mit Homöopathie zu tun. Hahnemann war begeistert von der damals neu eingeführten Pockenimpfung, die er als homöopathische Handlung und grossen medizinischen Fortschritt betrachtete.

Wingeier: Notwendig oder nicht ist eine sehr individuelle Entscheidung. Es kommt einerseits auf die Erkrankung und das Risiko an, andererseits auf die Einstellung und Risikobereitschaft der Eltern. Man muss vielmehr von den einzelnen Erkrankungen ausgehen, gegen die es eine Impfung gibt, als generell von Impfungen $\mathrm{zu}$ sprechen. Ich denke, gerade Tetanus ist eine wertvolle Impfung, und es braucht schon Mut und Überzeugung, sich nicht dagegen zu impfen. Wichtig ist, dass ein Impfentscheid individuell und frei erfolgen kann, ohne Angst, in voller Verantwortung. Dafür braucht es eine Auseinandersetzung mit der Erkrankung und der Impfung.

Schaller: Als Allgemeinarzt werde ich in der Impffrage nur beratend beigezogen. Ich empfehle die Basisimpfungen, d.h. DPT, Hib, Polio, MMR.

\section{Wie stehen Sie zu neuen Impfungen wie diejenige gegen humane Papillomaviren (HPV) oder die Schweinegrippe?}

Wingeier: Sehr kritisch. Gerade die sehr rasche Einführung der HPVImpfung unter Druck mit sehr schwacher Datenbasis hinterlässt ein sehr ungutes Gefühl. Das Theater mit der Schweinegrippe war eine traurige Angelegenheit.

Schaller: Persönlich bin ich skeptisch gegenüber der HPV-Impfung. Ich gehe davon aus, dass wir in einigen Jahren von den Epidemiologen und Gynäkologen mehr dazu erfahren werden. Bei den saisonalen Viruserkrankungen empfehle ich die Impfung nur für Risikopatienten.

Frei: Die HPV-Impfung wurde überstürzt eingeführt und ist deshalb auch auf höchster wissenschaftlicher Ebene umstritten. Bei der Schweinegrippe hatten wir einfach Glück: Der Erreger war derselbe wie bei der Spanischen Grippe 1918, die bei 28\% der Erkrankten tödlich verlief.

In der westlichen Gesellschaft nehmen Allergien stark zu, insbesondere bei Kindern: Neurodermitis, Heuschnupfen, Nahrungsmittelallergien usw. Wie behandeln Sie diese Probleme?

Schaller: Bei akuter und sehr starker Symptomatik verwende ich die chinesische Arzneitherapie zum Teil ergän- 


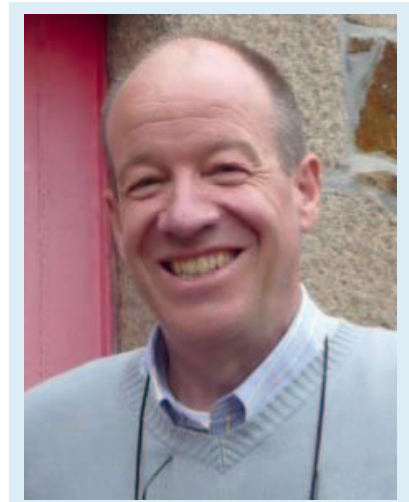

\section{Dr. med. Heiner Frei}

(heiner.frei@hin.ch) ist Kinderarzt FMH mit Schwerpunkt Homöopathie; seine Praxis ist in Laupen (BE). Unter seiner Federführung wurde die weltweit erste und bisher einzige Langzeitstudie über die homöopathische Behandlung bei ADHSKindern durchgeführt.

"Man muss die Impffrage unbedingt von der Homöopathie abkoppeln; Impfgegnerschaft hat per se nichts mit Homöopathie zu tun.»

zend zur schulmedizinischen Akuttherapie. Ansonsten behandle ich die allergischen Erkrankungen mit chinesischer Arzneitherapie, möglichst ohne immunsupprimierende Medikation.

Frei: Neurodermitis, Heuschnupfen und Nahrungsmittelallergien behandeln wir homöopathisch. Das geht in der Regel gut. Den akuten Asthmaanfall aber konventionell. Wir versuchen danach, das Asthma homöopathisch zu heilen, was nicht selten auch gelingt.

Wingeier: Weil den Krankheiten ein ähnliches Muster zugrunde liegt, gibt es einerseits eine gewisse Palette an Basisheilmitteln. Da aber nicht jeder Patient gleich ist, sondern seine eigene Geschichte mitbringt, muss man diese Basis individuell anpassen. Neben den Heilmitteln kommen dann je nach Fall noch Heileurythmie oder Sprachtherapie oder eine andere künstlerische Therapie ergänzend dazu. Jede
Behandlung einer solchen Krankheit ist ein Prozess und Weg; es geht um eine nachhaltige Gesundung und nicht alleine um die Behandlung der Symptome.

\section{Wie gehen Sie bei Mittelohrentzün-} dung und anderen bei Kindern häufigen Infektionen vor?

Wingeier: Ich sehe die Kinder, mache eine Anamnese und Befunderhebung, wenn notwendig weitere Diagnostik. In den meisten Fällen kann man eine Mittelohrentzündung ohne Antibiotika behandeln. Am häufigsten kommen Apis/Belladonna D3/D3 Glob, Levisticum Glob, homöopathisch abschwellende Nasentropfen und Zwiebelwickel zum Einsatz. Wichtig ist die Nachkontrolle. Man darf den Zeitpunkt nicht verpassen, bei dem man ein Antibiotikum einsetzen muss, weil es das Kind nicht alleine mit seinen Selbstheilungskräften schafft.

Schaller: Die schulmedizinische und Laboruntersuchung wird ergänzt durch TCM-Diagnostik. Falls meine Einschätzung und die Betreuungssituation des Kindes es erlauben, benütze ich ein Zeitfenster von 24 bis 36 Stunden für eine Behandlung mit TCM-Arzneitherapie. Oft erübrigt sich dadurch eine schulmedizinische Akuttherapie. Bei chronisch wiederkehrenden Entzündungen und Infekten verwende ich die an die Akutphase anschliessende Zeit, um über diätetische Massnahmen und Arzneitherapie mögliche konstitutionelle Kofaktoren zu verbessern.

Frei: Das konventionell-diagnostische und das homöopathische Vorgehen bei einer Mittelohrentzündung ist genau gleich. Aber das Reservemittel verabreichen wir schon nach 6 Stunden, falls das Kind dann noch Schmerzen hat. Sind diese nach 12 Stunden nicht verschwunden, so dürfen die Eltern ein Antibiotikum verabreichen, das sie ebenfalls als Reserve mitbekommen. $72 \%$ der Mittelohrentzündungen werden so ohne Antibiotika geheilt. Andere Infektionen werden gleich behandelt wie unter der vorhergehenden Frage beschrieben.

\section{Wie wirken Ihre Methoden bei ADHS?}

Frei: In der Langzeitbeobachtung über fünf Jahre gingen $75 \%$ aller Teilnehmer der Doppelblindstudie gut mit Homöopathie alleine. Sie hatten im Durchschnitt eine Abnahme der Symptomenintensität um $63 \%$.

Wingeier: Die Heilmittel und hier gerade die begleitenden und unterstützenden Massnahmen der Erziehung, das Krankheitsverständnis sowie Heileurythmie und künstlerische Therapien wirken in der Entwicklung unterstützend und aufbauend. In vielen Fällen gelingt es so, die Spitzen zu nehmen, dem Kind zu mehr Ruhe und Konzentration zu verhelfen. Dies ist aber von vielen Faktoren abhängig.

Schaller: Ich habe wenig Erfahrung in der TCM-Behandlung von ADHSKindern. Diese Behandlung überlasse ich dem Pädiater und dem Kinderpsychiater.

Verschreiben Sie auch Medikamente wie Ritalin, und wenn ja, unter welchen Bedingungen?

Frei: Ja, in Notfällen, wenn entweder in der Schule gar nichts mehr geht oder die Familie dem ADHS nicht mehr gewachsen ist. Insgesamt betrifft das etwa $10 \%$ unserer ADHS-Patienten.

Wingeier: Ja, wenn es nicht anders geht und wenn die Gefahr besteht, dass das Kind die sozialen Kontakte und die Integration in der Gesellschaft, Familie und Schule verliert. Wenn der Leidensdruck sehr hoch ist.

Interview: Irène Dietschi 